\title{
Evaluating the difference between autorefraction and subjective refraction may guide "fudge factor" for IOL power selection for cataract surgery after previous LASIK
}

\author{
Keith Ong ${ }^{1,2,3,4,5}$, Daisy Shu ${ }^{5,6}$ \\ 'Department of Ophthalmology, Northern Clinical School, University of Sydney, \\ Sydney, Australia; ${ }^{2}$ Department of Ophthalmology, Royal North Shore Hospital, \\ Sydney, Australia; ${ }^{3}$ Department of Ophthalmology, Sydney Adventist Hospital, \\ Sydney, Australia; ${ }^{4}$ Department of Ophthalmology, Chatswood Private Hospital, \\ Sydney, Australia; ${ }^{5}$ Discipline of Anatomy \& Histology, Bosch Institute, University \\ of Sydney, Australia
}

\begin{abstract}
We propose a method to estimate "fudge factor" in the selection of IOL power for post-myopic LASIK patients undergoing cataract surgery by considering the difference between subjective refraction (SR) and autorefraction (AR) and to also compare the predictability of two popular regression formulae: SRK-T and Haigis.
\end{abstract}

Keywords: autorefraction, cataract, IOL, LASIK, subjective refraction

\section{Introduction}

The challenges associated with calculating accurate intraocular lens (IOL) power in eyes after laser in situ keratomileusis (LASIK) have been well documented..$^{1-5}$ It is common for patients to experience hyperopic "refractive surprise" in post-myopic LASIK based on standard regression formulae for IOL power determination. ${ }^{3}$

This imprecision in IOL calculation is caused by the inability to accurately measure the corneal power using topography or keratometry. Corneal power is determined using a topography or keratometry, which assumes that the power of the cornea's paracentral 3 to $4 \mathrm{~mm}$ does not significantly differ from that of the central cornea. ${ }^{3}$ While this assumption is clinically acceptable for most normal eyes, it is no longer accurate for post-LASIK patients with abnormal corneal curvatures and results in inaccurate $\mathrm{IOL}$ power calculations.

Correspondence: Dr Keith Ong, 2 Railway Avenue, Eastwood, NSW 2122, Australia.

E-mail: keithong@optusnet.com.au 


\section{Report}

Many methods have been proposed for the determination of IOL power in post-LASIK patients with mixed success, due to variability of the amount of LASIK correction done and corneal curvature changes of the thinner cornea over time. In this small study, we propose a method to estimate "fudge factor" in the selection of IOL power for post-myopic LASIK patients undergoing cataract surgery by considering the difference between subjective refraction (SR) and autorefraction (AR) and to also compare the predictability of two popular regression formulae: SRK-T and Haigis. The Haigis-L formula was not used as it already had a fairly constant fudge factor built-in to compensate for the reduced central corneal curvature of the post-op myopic LASIK cornea.

A retrospective case analysis was conducted on 10 consecutive eyes of seven patients with history of LASIK for myopia who underwent phacoemulsification with implantation of Alcon SN60WF posterior chamber intraocular lens from 2007 to 2013. The difference between AR and SR was compared post-cataract surgery, and the predictability of SRK-T and Haigis IOL power calculation formulae was evaluated by comparing the post-cataract surgery SR and AR to the refraction predicted by the formulae. Zeiss IOL Master 500 (Carl Zeiss Meditec, Jena, Germany) was used for biometry and IOL power calculation. The autorefractor used was Topcon RM3300 (Topcon Corporation, Japan).

Post-cataract surgery AR was significantly more minus than SR (spherical equivalent $[S E] \pm S D, 0.60 \pm 0.44 D, p<0.05)$. The amount of LASIK correction showed a weak positive correlation with the difference between post-cataract surgery SR and $A R$, which was not statistically significant $(r=0.63)$. The difference between predicted refraction and post-cataract surgery AR was significantly less ( $p<$ $0.05)$ when calculated with Haigis $(-0.061 \pm 0.68 \mathrm{D})$ in comparison to SRK-T $(-0.65 \pm$ $0.66 \mathrm{D})$. There was also a significant difference $(p<0.05)$ between predicted refractions and post-cataract surgery SR when calculated with Haigis $(-0.54 \pm 0.66 \mathrm{D})$ in comparison to SRK-T $(-1.25 \pm 0.76 \mathrm{D})$.

$A R$ with the Topcon autorefractor yields a more minus refraction in comparison to SR following LASIK. Post-cataract surgery in these eyes, AR also yields a more minus refraction in comparison to $S R$ and the difference is fairly similar for a particular eye. Both SRK-T and Haigis formulae were more accurate in predicting post-cataract surgery AR than SR, and the difference between AR and SR is quite similar pre- and post-cataract surgery for a particular eye using the Topcon RM3300 autorefractor and Zeiss IOL Master 500. A different autorefractor and optical biometry instrument may yield a different fudge factor. Hence, this difference between AR and SR can be used as a guide for "fudge factor" when selecting the IOL power to achieve target SR. 


\section{References}

1. Vahid F, Mannis MJ, Garcia-Ferrer F, et al. Intraocular lens power calculation after laser in situ keratomileusis for myopia and hyperopia: a standardized approach. Cornea. 2001;20(8):792-797.

2. Randleman JB, Loupe DN, Song CD, Waring GO, Stulting RD. Intraocular lens power calculations after laser in situ keratomileusis. Cornea. 2002 Nov;21(8):751-755.

3. Makool RJ, Ko W, Mackool R. Intraocular lens power calculation after laser in situ keratomileusis. Aphakic refraction technique. J Cataract Refract Surg. 2006 March;32(3):435-437.

4. Walter KA, Gagnon MR, Hoopes PC, Dickinson PJ. Accurate intraocular lens power calculation after myopic laser in situ keratomileusis, bypassing corneal power. J Cataract Refract Surg. 2006 Mar;32(3):425-429.

5. Shammas HJ, Shammas MC. No-history method of intraocular lens power calculation for cataract surgery after myopic laser in situ keratomileusis. J Cataract Refract Surg. 2007 Jan;33(1):31-36. 